\title{
The Cluster Expansion for the Self-Gravitating gas and the Thermodynamic Limit
}

\author{
H. J. de $\operatorname{Vega}^{(a)}$ and N. G. Sánchez ${ }^{(b)}$ \\ (a) Laboratoire de Physique Théorique et Hautes Energies, \\ Université Paris VI et VII, Tour 16, 1er étage, 4, Place Jussieu, \\ 75252 Paris, cedex 05, FRANCE. Laboratoire Associé au CNRS UMR 7589. and \\ (b) Observatoire de Paris, LERMA, \\ 61, Avenue de l'Observatoire, 75014 Paris, FRANCE. \\ Laboratoire Associé au CNRS UMR 8112.
}

(Dated: May 30, 2018)

\begin{abstract}
We develop the cluster expansion and the Mayer expansion for the self-gravitating thermal gas and prove the existence and stability of the thermodynamic limit $N, V \rightarrow \infty$ with $N / V^{\frac{1}{3}}$ fixed. The essential (dimensionless) variable is here $\eta \equiv \frac{G m^{2} N}{V^{\frac{1}{3}} T}$ (which is kept fixed in the thermodynamic limit). We succeed in this way to obtain the expansion of the grand canonical partition function in powers of the fugacity. The corresponding cluster coefficients behave in the thermodynamic limit as $\left(\frac{\eta}{N}\right)^{j-1} c_{j}$ where $c_{j}$ are pure numbers. They are expressed as integrals associated to tree cluster diagrams. A bilinear recurrence relation for the coefficients $c_{j}$ is obtained from the mean field equations in the Abel's form. In this way the large $j$ behaviour of the $c_{j}$ is calculated. This large $j$ behaviour provides the position of the nearest singularity which corresponds to the critical point (collapse) of the self-gravitating gas in the grand canonical ensemble. Finally, we discuss why other attempts to define a thermodynamic limit for the self-gravitating gas fail.
\end{abstract}

Contents

I. Introduction 1

II. The Cluster Expansion for the Self-Gravitating Gas

III. The Maver Expansion for the self-gravitating gas 6

IV. The Grand Canonical Ensemble for the self-gravitating gas 8

V. Calculation of the Cluster coefficients $c_{n}$

VI. The stability of the thermodvnamic limit $\mathbf{N} \cdot \mathbf{V}=\mathbf{L}^{\mathbf{3}} \rightarrow \infty$ with $\frac{\mathbf{N}}{\mathrm{I}}$ fixed (fixed $\eta$ )

References 13

\section{INTRODUCTION}

The self-gravitating gas has been the subject of attention since many years [1, 2, 3, 4, 5]. In refs. 2, 3] we recently investigated the self-gravitating thermal gas using Monte Carlo simulations, mean field methods and low density expansions [2, 3]. We have shown that the system possess a well defined infinite volume limit in the grand canonical (GC), the canonical (C) and microcanonical (MC) ensembles when $N, V \rightarrow \infty$ keeping $N / V^{\frac{1}{3}}$ fixed. A relevant variable here is dimensionless ratio

$$
\eta \equiv \frac{G m^{2} N}{V^{\frac{1}{3}} T}
$$

which is kept fixed in the $N, V \rightarrow \infty$ limit. All physical quantities per particle turn out to be functions of the single variable $\eta$ and are well defined and finite in the thermodynamic limit $N, V \rightarrow \infty$ with $\eta$ fixed.

In this paper we develop the cluster expansion and Mayer's approach to the self-gravitating thermal gas and provide a rigorous demonstration of the existence of the thermodynamic limit $N, V \rightarrow \infty$ with $\eta$ fixed.

The cluster expansion is a powerful tool allowing to express the partition function in a power series of the density for short range interactions 6]. We apply and adapt this method (the Mayer expansion) which is purely combinatorial to the self-gravitating gas. We succeed in this way to obtain the coordinate partition function $Q_{N}$ as a power series 
in the thermodynamic limit. This is derived by generalizing the saddle point method used in ref. [6]. The expansion is obtained in terms of the coefficients $c_{n}$ which are pure numbers. More explicitly,

$$
\frac{1}{N} \log Q_{N}(\eta) \stackrel{N \gg 1}{=} \frac{1}{\eta} g\left(\eta t_{\eta}\right)-\log t_{\eta}-1+\mathcal{O}\left(\frac{1}{N}\right) .
$$

where

$$
g(x) \equiv \sum_{j=1}^{+\infty} c_{j} x^{j}
$$

and $t_{\eta}$ is the solution of the equation,

$$
t_{\eta} g^{\prime}\left(t_{\eta} \eta\right)=1 \quad, \quad \text { i. e. } \quad \sum_{j=1}^{+\infty} j c_{j}\left(t_{\eta} \eta\right)^{j}=\eta
$$

Moreover, $-\frac{1}{N} \log Q_{N}(\eta)$ is the free energy of the self-gravitating gas minus the free energy of an ideal gas divided by $N T$. The series for $g\left(\eta t_{\eta}\right)$ in eqs.(1.2)-(1.3) is therefore a high temperature or low density expansion [see eq.(1.1)].

The coefficients $c_{n}$ can be expressed in the thermodynamic limit as a sum of $3 n$-uple integrals associated to tree cluster diagrams. Loop cluster diagrams are subdominant for $N \rightarrow \infty$. The coefficients $c_{n}$ only depend on the geometry of the box. The first $c_{n}$ are obtained by explicit evaluation of the cluster integrals. We have for the sphere,

$$
c_{1}=1 \quad, \quad c_{2}^{\text {sphere }}=\frac{3}{5}\left(\frac{4 \pi}{3}\right)^{1 / 3} \quad, \quad c_{3}^{\text {sphere }}=\frac{51}{70}\left(\frac{4 \pi}{3}\right)^{2 / 3} \quad, \quad c_{4}^{\text {sphere }}=\frac{4 \pi}{3} \frac{373}{315} .
$$

Moreover, we use the connection with the mean field approach to obtain a nonlinear recurrence relation for the coefficients $c_{n}^{\text {sphere }}$ :

$$
c_{n}^{R}=\frac{1}{(2 n+1)(n-1)} \sum_{s=1}^{n-1} c_{s}^{R} c_{n-s}^{R} s^{2}(2 n-2 s+1) \quad \text { for } \quad n \geq 2,
$$

where

$$
c_{n}^{\text {sphere }}=\left(\frac{4 \pi}{3}\right)^{\frac{n-1}{3}} c_{n}^{R} .
$$

(The label $R$ stands for the spherical geometry). This allows to compute systematically all $c_{n}^{R}$ and to find their large $n$ behaviour as

$$
c_{n}^{R} \stackrel{n \geqq 1}{=} 0.309360 \ldots \frac{n^{-\frac{5}{2}}}{\left(u_{G C}\right)^{n}}\left[1+\mathcal{O}\left(\frac{1}{n}\right)\right] .
$$

Here, $u_{G C}=0.30034 \ldots$ is the radius of convergence of the series eq.(1.3). Therefore, $u=u_{G C}$ is the nearest singularity of $g_{R}(u)$ in the $u$-plane. by

We show below that the function $g$ is related to the partition function $\mathcal{Z}_{G C}(\eta, z)$ in the grand canonical ensemble

$$
\log \mathcal{Z}_{G C}(\eta, z)=\frac{N}{\eta} g\left(\eta t_{\eta}\right)
$$

where

$$
\mathcal{Z}_{G C}(\eta, z)=\sum_{N=0}^{+\infty} \frac{Q_{N}(\eta)}{N !}\left[\left(\frac{m T}{2 \pi}\right)^{\frac{3}{2}} V z\right]^{N}
$$

In addition, the fugacity $\left(z=e^{\frac{\mu}{T}}\right)$ turns out to be given by $z=t_{\eta} e^{\frac{\mu_{0}}{T}}$, where $\mu$ and $\mu_{0}$ are the chemical potentials for the self-gravitating gas and for the ideal gas, respectively. 
The point $u_{G C}=0.30034 \ldots$ where $g(u)$ is singular corresponds to the critical point (collapse) in the grand canonical ensemble. Recall that the collapse point depends on the ensemble considered [2].

In conclusion, our investigation here on the cluster expansion and the Mayer expansion for the self-gravitating gas shows that:

- The self-gravitating gas admits a consistent thermodynamic limit $N, V \rightarrow \infty$ with $\frac{N}{V^{\frac{1}{3}}}$ fixed. In this limit, extensive thermodynamic quantities like energy, free energy, entropy are proportional to $N$. That is, in this limit the energy, free energy, and entropy per particle are well defined and finite.

- The cluster expansion and the mean field approach provide the same results in the thermodynamic limit $N, V \rightarrow \infty$ with $\frac{N}{V^{\frac{1}{3}}}$ fixed.

- The partition function needs a small short-distance cutoff $a$ in order to be well defined, the thermodynamic limit has a finite limit for $a \rightarrow 0$. In other words, the contributions to the partition function which diverge for $a \rightarrow 0$ are subdominant for $N \rightarrow \infty$ (see sec. II). For large $N$ and fixed cutoff $a$ the potentially divergent contributions for $a \rightarrow 0$ are suppresed by a factor at least $\eta^{2} / N^{2}$ compared with the dominant contribution for $N \rightarrow \infty$. That is, the $N, V=\infty$ limit of the cutoff model (with $\frac{N}{V^{\frac{1}{3}}}$ fixed) has a finite limit for $a \rightarrow 0$.

Therefore, subdominant corrections in $\eta / N$ can always be neglected. Realistic models of the self-gravitating gas (interstellar medium, galaxy distribution) require a small non-zero short distance cutoff since molecular and atomic forces dominate over gravitational forces for short distances.

\section{THE CLUSTER EXPANSION FOR THE SELF-GRAVITATING GAS}

We investigate in this section the self-gravitating gas in thermal equilibrium at temperature $T \equiv \beta^{-1}$. That is, we work in the canonical ensemble where the system of $N$ particles is in contact with a thermal bath at temperature $T$. We assume the gas being on a cubic box of side $L$.

The partition function of the system can be written as

$$
\mathcal{Z}_{C}(N, T)=\frac{1}{N !} \int \ldots \int \prod_{l=1}^{N} \frac{d^{3} p_{l} d^{3} q_{l}}{(2 \pi)^{3}} e^{-\beta H_{N}}
$$

where

$$
H_{N}=\sum_{l=1}^{N} \frac{p_{l}^{2}}{2 m}-G m^{2} \sum_{1 \leq l<j \leq N} \frac{1}{\left|\vec{q}_{l}-\vec{q}_{j}\right|_{A}}
$$

$G$ is Newton's gravitational constant.

At short distances, the particle interaction for the self-gravitating gas in physical situations is not gravitational. Its exact nature depends on the problem under consideration (opacity limit, Van der Waals forces for molecules etc.). We shall just assume a repulsive short distance potential, that is,

$$
v_{A}\left(\left|\vec{q}_{l}-\vec{q}_{j}\right|\right)=-\frac{1}{\left|\vec{q}_{l}-\vec{q}_{j}\right|_{A}}=\left\{\begin{array}{cc}
-\frac{1}{\left|\vec{q}_{l}-\vec{q}_{j}\right|} & \text { for }\left|\vec{q}_{l}-\vec{q}_{j}\right| \geq A \\
+\frac{1}{A} & \text { for }\left|\vec{q}_{l}-\vec{q}_{j}\right| \leq A
\end{array}\right.
$$

where $A<<L$ is the short distance cut-off.

The integrals over the momenta $p_{l},(1 \leq l \leq N)$ in eq. 2.1) can be computed immediately.

It is convenient to introduce the dimensionless variables $\vec{r}_{l}, 1 \leq l \leq N$ making explicit the volume dependence as

$$
\begin{aligned}
\vec{q}_{l} & =L \vec{r}_{l}, \quad \vec{r}_{l}=\left(x_{l}, y_{l}, z_{l}\right) \\
0 & \leq x_{l}, y_{l}, z_{l} \leq 1 .
\end{aligned}
$$

That is, in the new coordinates the gas is inside a cube of unit volume. 
The partition function takes now the form,

$$
\mathcal{Z}_{C}(N, T)=\frac{1}{N !}\left(\frac{m T L^{2}}{2 \pi}\right)^{\frac{3 N}{2}} Q_{N}(\eta)
$$

where

$$
Q_{N}(\eta) \equiv \int_{0}^{1} \ldots \int_{0}^{1} \prod_{l=1}^{N} d^{3} r_{l} e^{\eta u\left(\vec{r}_{1}, \ldots, \vec{r}_{N}\right)}
$$

$\eta$ is the dimensionless variable [2]

$$
\eta \equiv \frac{G m^{2} N}{L T}
$$

and $u\left(\vec{r}_{1}, \ldots, \vec{r}_{N}\right)$ is defined by

$$
u\left(\vec{r}_{1}, \ldots, \vec{r}_{N}\right) \equiv \frac{1}{N} \sum_{1 \leq l<j \leq N} \frac{1}{\left|\vec{r}_{l}-\vec{r}_{j}\right|_{a}} \quad, \quad a \equiv A / L \ll 1
$$

In this way all dependence on the volume $V=L^{3}$ is buried in the variable $\eta$.

The coordinate partition function $Q_{N}(\eta)$ can be written as

$$
Q_{N}(\eta)=\int_{0}^{1} \cdots \int_{0}^{1} \prod_{l=1}^{N} d^{3} r_{l} \prod_{1 \leq l<j \leq N} e^{\frac{\eta}{N\left|\vec{r}_{l}-\vec{r}_{j}\right| a}}
$$

We are now interested to expand $Q_{N}(\eta)$ in powers of $\frac{\eta}{N}$. In order to do this is convenient to define:

$$
f_{l j} \equiv e^{\frac{\eta}{N\left|\vec{r}_{l}-\vec{r}_{j}\right| a}}-1
$$

For small $\frac{\eta}{N}$ and fixed $a>0$ we have,

$$
f_{l j}=\frac{\eta}{N\left|\vec{r}_{l}-\vec{r}_{j}\right|_{a}}+\mathcal{O}\left[\left(\frac{\eta}{N}\right)^{2}\right]
$$

All the integrals over $r_{l}$ in eq.(2.6) are finite provided we keep $a>0$.

We can now multiply out the products of $f$ 's in the coordinate partition function $Q_{N}(\eta)$,

$$
\prod_{1 \leq l<j \leq N}\left(1+f_{l j}\right)=1+\sum f_{i j}+\sum f_{i j} f_{k l}+\ldots
$$

Thus, by introducing the $f_{i j}$ functions the effects of interparticle forces are better exhibited.

A systematic treatment of such sum of products can be found in ref. [6] . The outcome is that a general term in the partition function $Q_{N}(\eta)$ eq.(2.9), can be factorized as the product of several integrals over the coordinates $\vec{r}_{j}$. Each integral corresponds to a 'cluster' of particles and is called $b_{j}(\eta, N)$ where

$$
b_{j}(\eta, N)=\frac{1}{j !} \iint_{0}^{1} \ldots \int_{0}^{1} \prod_{l=1}^{j} d^{3} r_{l} S_{1,2, \ldots, j},
$$

with [6],

$$
\begin{aligned}
& S_{j}=1 \\
& S_{12}=f_{12} \\
& S_{123}=f_{12} f_{23}+f_{12} f_{13}+f_{13} f_{23}+f_{12} f_{13} f_{23}
\end{aligned}
$$




$$
\begin{aligned}
& S_{1234}=f_{12} f_{23} f_{34}+\text { nineteen permutations+ } \\
& +f_{12} f_{13} f_{24} f_{34}+\text { fourteen permutations }+ \\
& +f_{12} f_{13} f_{14} f_{24} f_{34}+\text { five permutations }+f_{12} f_{13} f_{14} f_{23} f_{24} f_{34} .
\end{aligned}
$$

The main result is that $Q_{N}(\eta)$ can be expressed as the infinite sum, the so called 'cluster expansion' as:

$$
Q_{N}(\eta)=N ! \sum_{\tilde{m}, \sum_{j=1}^{N}\left(j m_{j}\right)=N} \prod_{j=1}^{N} \frac{\left[b_{j}(\eta, N)\right]^{m_{j}}}{m_{j} !}
$$

where

$$
\widetilde{m} \equiv\left(m_{1}, \ldots, m_{N}\right)
$$

It must be stressed that eqs.(2.11)-(2.13) are purely combinatorial and they apply both for the short range interactions considered in ref. [6] as well as the long range Newton potential.

Since $f_{l j}=\mathcal{O}\left(\frac{\eta}{N}\right)$ for large $N$, and $S_{1,2, \ldots, j}$ contains at least a product of $j$ factors $f_{i l}$, we see that

$$
S_{1,2, \ldots, j}=\mathcal{O}\left[\left(\frac{\eta}{N}\right)^{j-1}\right] \quad, \quad \text { for } \quad N \gg j
$$

Therefore, the cluster integrals eq.(2.11) take the form

$$
b_{j}(\eta, N)=\left(\frac{\eta}{N}\right)^{j-1} c_{j}\left[1+\mathcal{O}\left(\frac{\eta}{N}\right)\right] \quad, \quad \text { for } \quad N \gg j .
$$

Here the coefficients $c_{j}$ are positive numbers which only depend on the geometry of the box. As shown in fig. 1 the dominant terms for large $N$ are cluster diagrams with a tree structure. In the large $N$ limit, cluster diagrams with a loop structure as the last term in eq.(2.12) are subdominant.

From eqs.(2.10) $-(2.12)$ and (2.14) we find,

$$
\begin{aligned}
& c_{1}=1 \\
& c_{2}=\frac{1}{2} \int_{0}^{1} \int_{0}^{1} \frac{d^{3} r_{1} d^{3} r_{2}}{\left|\vec{r}_{1}-\vec{r}_{2}\right|} \\
& c_{3}=\frac{1}{2} \int_{0}^{1} \int_{0}^{1} \int_{0}^{1} \frac{d^{3} r_{1} d^{3} r_{2} d^{3} r_{3}}{\left|\vec{r}_{1}-\vec{r}_{2}\right|\left|\vec{r}_{2}-\vec{r}_{3}\right|} .
\end{aligned}
$$

For a sphere of unit volume we find [2]

$$
c_{2}^{\text {sphere }}=\frac{3}{5}\left(\frac{4 \pi}{3}\right)^{1 / 3}=0.967195171 \ldots \quad, \quad c_{3}^{\text {sphere }}=\frac{51}{70}\left(\frac{4 \pi}{3}\right)^{2 / 3}=1.893206013 \ldots
$$

For the cubic geometry chosen, it takes the value [2]

$$
c_{2}^{\text {cube }}=4 \int_{0}^{1}(1-x) d x \int_{0}^{1}(1-y) d y \int_{0}^{1} \frac{(1-z) d z}{\sqrt{x^{2}+y^{2}+z^{2}}}=0.94116 \ldots
$$

Furthermore, we find for the coefficient $c_{4}$,

$$
c_{4}=\frac{1}{2} \int_{0}^{1} \int_{0}^{1} \int_{0}^{1} \int_{0}^{1} \frac{d^{3} r_{1} d^{3} r_{2} d^{3} r_{3} d^{3} r_{4}}{\left|\vec{r}_{1}-\vec{r}_{2}\right|\left|\vec{r}_{2}-\vec{r}_{3}\right|\left|\vec{r}_{3}-\vec{r}_{4}\right|}+\frac{1}{6} \int_{0}^{1} \int_{0}^{1} \int_{0}^{1} \int_{0}^{1} \frac{d^{3} r_{1} d^{3} r_{2} d^{3} r_{3} d^{3} r_{4}}{\left|\overrightarrow{r_{1}}-\overrightarrow{r_{2}}\right|\left|\vec{r}_{2}-\vec{r}_{3}\right|\left|\vec{r}_{2}-\vec{r}_{4}\right|} .
$$

The combinatorial factors $\frac{1}{2}$ and $\frac{1}{6}$ take into account the symmetries of the respective cluster diagrams. 
Inserting here the expansion in spherical harmonics,

$$
\frac{1}{\left|\vec{r}-\vec{r}^{\prime}\right|}=4 \pi \sum_{l=0}^{\infty} \frac{1}{2 l+1} \frac{r_{<}^{l}}{r_{>}^{l+1}} \sum_{m=-l}^{m=+l} \bar{Y}_{l m}\left(\hat{r}^{\prime}\right) Y_{l m}(\hat{r}),
$$

where $r_{>} \equiv \max \left(r, r^{\prime}\right)$ and $r_{<} \equiv \min \left(r, r^{\prime}\right)$, the angular integrals and then the radial integrals can be performed with the result,

$$
\int_{0}^{1} \int_{0}^{1} \int_{0}^{1} \int_{0}^{1} \frac{d^{3} r_{1} d^{3} r_{2} d^{3} r_{3} d^{3} r_{4}}{\left|\vec{r}_{1}-\vec{r}_{2}\right|\left|\vec{r}_{2}-\vec{r}_{3}\right|\left|\vec{r}_{3}-\vec{r}_{4}\right|}=(4 \pi)^{4} \int_{0}^{b} \ldots \int_{0}^{b} \frac{\prod_{l=1}^{4} r_{l}^{2} d r_{l}}{r_{1,2}^{>} r_{2,3}^{>} r_{3,4}^{>}}=\frac{4 \pi}{3} \frac{62}{35}
$$

and

$$
\int_{0}^{1} \int_{0}^{1} \int_{0}^{1} \int_{0}^{1} \frac{d^{3} r_{1} d^{3} r_{2} d^{3} r_{3} d^{3} r_{4}}{\left|\vec{r}_{1}-\vec{r}_{2}\right|\left|\vec{r}_{2}-\vec{r}_{3}\right|\left|\vec{r}_{2}-\vec{r}_{4}\right|}=(4 \pi)^{4} \int_{0}^{b} \ldots \int_{0}^{b} \frac{\prod_{l=1}^{4} r_{l}^{2} d r_{l}}{r_{1,2}^{>} r_{2,3}^{>} r_{2,4}^{>}}=\frac{4 \pi}{3} \frac{188}{105}
$$

Therefore,

$$
c_{4}^{\text {sphere }}=\frac{4 \pi}{3} \frac{373}{315} .
$$

Notice that all these 'tree' cluster diagrams eqs.(2.15) and (2.17) have a finite limit for zero cutoff $a$.

Divergent pieces for $a \rightarrow 0$ are subdominant as $\frac{\eta^{2}}{N^{2}}$ for large $N$. The leading divergent contribution for $a \rightarrow 0$ to $Q_{N}(\eta)$ to the nth. order in $\eta$ takes the form [2]

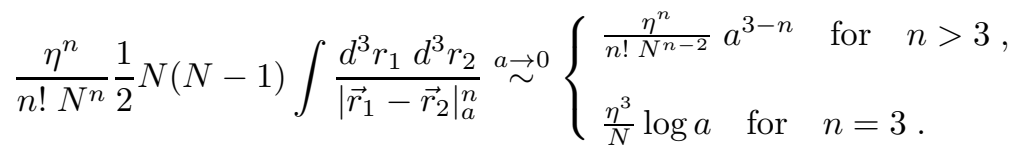

This gives for the physical quantities (see next section) contributions of the order

$$
\frac{\eta^{n}}{n ! N^{n-1}} a^{3-n} \text { for } n>3 \text { and } \frac{\eta^{3}}{N^{2}} \log a \text { for } n=3 .
$$

These contributions are clearly negligible in the $N \rightarrow \infty$ limit with fixed short-distance cutoff.

\section{THE MAYER EXPANSION FOR THE SELF-GRAVITATING GAS}

It is convenient to consider the generating function [6]

$$
X(\eta, z) \equiv \sum_{j=1}^{+\infty} b_{j}(\eta, N) z^{j}
$$

It can be shown that $[\underline{6}]$

$$
\sum_{N=0}^{+\infty} \frac{Q_{N}(\eta)}{N !} z^{N}=e^{X(\eta, z)}
$$

where $z$ is an auxiliary variable whose physical meaning (the fugacity) will appear in sec. IV.

Hence, we can compute the coefficients $Q_{N}(\eta)$ from eq.(3.2) by contour integration,

$$
\frac{Q_{N}(\eta)}{N !}=\oint \frac{d z}{2 \pi i} \frac{e^{X(\eta, z)}}{z^{N+1}} .
$$

We choose as contour a cercle of radius $r$,

$$
z=r e^{i \theta} \quad, \quad 0 \leq \theta \leq 2 \pi
$$


Integrating over $\theta$ yields,

$$
\frac{Q_{N}(\eta)}{N !}=\int_{-\pi}^{+\pi} \frac{d \theta}{2 \pi} e^{X\left(\eta, r e^{i \theta}\right)-N \log r-i N \theta}
$$

For large $N$, we can use eqs.(2.14) to express the $b_{j}(\eta, N)$ in $X(\eta, z)$ [see eq.(3.1)] and we find,

$$
X(\eta, z) \stackrel{N \geqq 1}{=} \frac{N}{\eta} \sum_{j=1}^{+\infty} c_{j}\left(\frac{z \eta}{N}\right)^{j}=\frac{N}{\eta} g\left(\frac{z \eta}{N}\right)
$$

where

$$
g(x) \equiv \sum_{j=1}^{+\infty} c_{j} x^{j}
$$

Thus,

$$
\frac{Q_{N}(\eta)}{N !} \stackrel{N \gg 1}{=} \int_{-\pi}^{+\pi} \frac{d \theta}{2 \pi} e^{N\left[\frac{1}{\eta} g\left(\frac{r e^{i \theta} \eta}{N}\right)-\log r-i \theta\right]}=\int_{-\pi}^{+\pi} \frac{d \theta}{2 \pi} e^{N \Psi(r, \theta)} .
$$

We can now apply the steepest descent method to this integral for large $N$ since the integrand has the structure $e^{N \Psi(r, \theta)}$ where,

$$
\begin{aligned}
& \Psi(r, \theta)=\frac{1}{\eta} g\left(\frac{r e^{i \theta} \eta}{N}\right)-\log r-i \theta \\
& \frac{\partial \Psi}{\partial \theta}(r, \theta)=i \frac{r e^{i \theta}}{N} g^{\prime}\left(\frac{r e^{i \theta} \eta}{N}\right)-i, \\
& \frac{\partial \Psi}{\partial r}(r, \theta)=\frac{e^{i \theta}}{N} g^{\prime}\left(\frac{r e^{i \theta} \eta}{N}\right)-\frac{1}{r}
\end{aligned}
$$

The saddle point, solution of $\frac{\partial \Psi}{\partial \theta}(r, \theta)=0=\frac{\partial \Psi}{\partial r}(r, \theta)$ is thus found at

$$
(r, \theta)_{\text {saddle }}=(N t, 0)
$$

where $t$ is $N$-independent and is a solution of the equation,

$$
t g^{\prime}(t \eta)=1
$$

That is, $t$ is a function of $\eta$ defined by the constraint eq.3.7 or more explicitly,

$$
\sum_{j=1}^{+\infty} j c_{j}(t \eta)^{j}=\eta
$$

Choosing $r=N t$ as integration path in eq. (3.5) and expanding the integrand around $\theta=0$ yields,

$$
\frac{Q_{N}(\eta)}{N !} \stackrel{N \geqq 1}{=} e^{N \Psi(N t, 0)} \int_{-\infty}^{+\infty} \frac{d \theta}{2 \pi} e^{-\frac{1}{2} N \theta^{2} \xi(\eta)}=\frac{e^{N \Psi(N t, 0)}}{\sqrt{2 \pi N \xi(\eta)}}\left[1+\mathcal{O}\left(\frac{1}{N}\right)\right]
$$

where,

$$
\begin{aligned}
& \Psi(N t, 0)=\frac{1}{\eta} g\left(\eta t_{\eta}\right)-\log \left[N t_{\eta}\right] \\
& \xi(\eta) \equiv-\frac{\partial^{2} \Psi}{\partial \theta^{2}}(N t, 0)=\sum_{j=1}^{+\infty} j^{2} c_{j} t^{j}>0
\end{aligned}
$$

where $t_{\eta}$ is a function of $\eta$ defined by eq.(3.7). 
Using now Stirling's formula for the $N$ ! factorial we find,

$$
\frac{1}{N} \log Q_{N}(\eta)=\frac{1}{\eta} g\left(\eta t_{\eta}\right)-\log t_{\eta}-1+\mathcal{O}\left(\frac{1}{N}\right)
$$

Therefore, the free energy can be written as,

$$
\frac{F-F_{0}}{N T}=-\frac{1}{\eta} g\left(\eta t_{\eta}\right)+\log t_{\eta}+1
$$

where $F_{0}$ stands for the free energy of the ideal gas,

$$
F_{0}=-N T \log \left[\frac{e V}{N}\left(\frac{m T}{2 \pi}\right)^{\frac{3}{2}}\right]
$$

The pressure (at the surface) follows from the thermodynamic relation

$$
p=-\left(\frac{\partial F}{\partial V}\right)_{T}
$$

we find

$$
f(\eta) \equiv \frac{p V}{N T}=\frac{2}{3}+\frac{g\left(\eta t_{\eta}\right)}{3 \eta} \text { and } \quad \frac{1}{N} \log Q_{N}(\eta)=3 \int_{0}^{\eta} d x \frac{1-f(x)}{x}
$$

The dimensionless ratio $\frac{p V}{N T}$ was called $f(\eta)$ in refs. 2, 3]. We can express all physical quantities in terms of the function $f(\eta)$. We find from eqs.(3.9) and (3.11),

$$
3 f(\eta)=2+\frac{g\left(\eta t_{\eta}\right)}{\eta} \text { and }-\frac{1}{\eta} g\left(\eta t_{\eta}\right)+\log t_{\eta}+1=-3 \int_{0}^{\eta} d x \frac{1-f(x)}{x} .
$$

That is,

$$
\frac{1}{3} \log t_{\eta}=f(\eta)-1-\int_{0}^{\eta} d x \frac{1-f(x)}{x} .
$$

We can solve eq.(3.7) for $t_{\eta}$ in powers of $\eta$ with the result,

$$
\begin{aligned}
& g\left(\eta t_{\eta}\right)=\eta t_{\eta}+c_{2}\left(\eta t_{\eta}\right)^{2}+c_{3}\left(\eta t_{\eta}\right)^{3}+\mathcal{O}\left(\eta^{4}\right) \\
& t_{\eta}=1-2 c_{2} \eta+\left[8\left(c_{2}\right)^{2}-3 c_{3}\right] \eta^{2}+\mathcal{O}\left(\eta^{3}\right) .
\end{aligned}
$$

and from eq. (3.12) we find for $f(\eta)$ and $g\left(\eta t_{\eta}\right)$,

$$
\begin{aligned}
& f(\eta)=1-\frac{c_{2}}{3} \eta+\frac{2}{3}\left[2\left(c_{2}\right)^{2}-c_{3}\right] \eta^{2}+\mathcal{O}\left(\eta^{3}\right) \\
& -3 \int_{0}^{\eta} d x \frac{1-f(x)}{x}=-c_{2} \eta+\left[2\left(c_{2}\right)^{2}-c_{3}\right] \eta^{2}+\mathcal{O}\left(\eta^{3}\right) \\
& g\left(\eta t_{\eta}\right)=\eta\left\{1-c_{2} \eta+2\left[2\left(c_{2}\right)^{2}-c_{3}\right] \eta^{2}\right\}+\mathcal{O}\left(\eta^{4}\right) .
\end{aligned}
$$

It must be noticed that the function $f(\eta)$ (and hence all physical quantities) have the same expression whether we compute it from the mean field approach [2, 3] or from the saddle point eqs. (3.5), (3.9) and (3.11).

\section{THE GRAND CANONICAL ENSEMBLE FOR THE SELF-GRAVITATING GAS}

The definition of the variable $z$ through eq.(3.2) suggests that $z$ is related to the fugacity. To be more precise, the grand partition function is defined in terms of the canonical partition function as,

$$
\mathcal{Z}_{G C}(\eta, z)=\sum_{N=0}^{+\infty} \mathcal{Z}_{C}(N, T) z^{N}=\sum_{N=0}^{+\infty} \frac{Q_{N}(\eta)}{N !}\left[\left(\frac{m T}{2 \pi}\right)^{\frac{3}{2}} V z\right]^{N}
$$


where we used eq.(2.5).

Therefore, $z$ must be multiplied by the ideal gas factor $\left(\frac{m T}{2 \pi}\right)^{\frac{3}{2}} V$.

At the saddle point we have after this renormalization

$$
\left(\frac{m T}{2 \pi}\right)^{\frac{3}{2}} V z=N t_{\eta} \quad \text { and } \quad X\left(\eta,\left(\frac{m T}{2 \pi}\right)^{\frac{3}{2}} V z\right)=\log \mathcal{Z}_{G C}(\eta, z) .
$$

Now, we know from ref. 2] that the chemical potential $\mu$ takes the form

$$
\frac{\mu-\mu_{0}}{T}=-3 \int_{0}^{\eta} d x \frac{1-f(x)}{x}-3[1-f(\eta)]
$$

where $\mu_{0}$ stands for the chemical potential of the ideal gas,

$$
\mu_{0}=-T \log \left[\frac{V}{N}\left(\frac{m T}{2 \pi}\right)^{\frac{3}{2}}\right] .
$$

From eq. (3.13) and 4.3) we have,

$$
\frac{\mu-\mu_{0}}{T}=\log t_{\eta}
$$

Therefore, from eqs.(4.2)- (4.5),

$$
t_{\eta}=e^{-\frac{\mu_{0}}{T}} z \quad, \quad e^{\frac{\mu}{T}}=z
$$

that is, we can identify $z$ with the fugacity.

We have found in ref. [2] that $f(\eta)$ obeys in the spherical case the first order differential equation of Abel's type,

$$
\eta^{R}\left[3 f\left(\eta^{R}\right)-1\right] f^{\prime}\left(\eta^{R}\right)+\left[3 f\left(\eta^{R}\right)-3+\eta^{R}\right] f\left(\eta^{R}\right)=0 .
$$

where the variable $\eta^{R}$ is defined as,

$$
\eta^{R} \equiv \eta\left(\frac{4 \pi}{3}\right)^{1 / 3}=1.61199 \ldots \eta
$$

Using eqs. (4.7) and (3.13) yields,

$$
\log t_{\eta}=\log f\left(\eta^{R}\right)-\eta^{R} \quad, \quad \text { i. e. } \quad t_{\eta}=e^{-\eta^{R}} f\left(\eta^{R}\right) .
$$

Eq. (4.1) provides the grand partition function. The above results showing that $\mathcal{Z}_{G C}(\eta, z)$ is dominated by the canonical ensemble, together with eq.(4.6) prove that the canonical and grand canonical ensembles are equivalent in their common region of validity as stated in refs. [2, 3].

In the thermodynamic limit, $\mathcal{Z}_{G C}(\eta, z)$ is given by eqs.(3.3) and (4.2) as

$$
\log \mathcal{Z}_{G C}(\eta, z)=\frac{N}{\eta} g\left(\frac{z \eta}{N}\left(\frac{m T}{2 \pi}\right)^{\frac{3}{2}} V\right)=\frac{N}{\eta} g\left(\eta t_{\eta}\right) .
$$

Using here eq.(3.12) gives,

$$
\log \mathcal{Z}_{G C}(\eta, z)=N[3 f(\eta)-2],
$$

which exactly coincides with the expression found in ref. [2] for the grand partition function in the mean field approach. 


\section{CALCULATION OF THE CLUSTER COEFFICIENTS $c_{n}$}

We compute in this section the cluster coefficients $c_{n}$ for the sphere by using a non-linear differential equation for the function $g(u)$.

We first find from eq.(3.7)

$$
u g_{R}^{\prime}(u)=\eta^{R} \quad \text { with } \quad u \equiv t_{\eta} \eta^{R} \quad \text { and } \quad g_{R}(u) \equiv\left(\frac{4 \pi}{3}\right)^{1 / 3} g(u)
$$

Then, combining eqs.(4.7) and (5.1) yields the second order differential equation for $g_{R}(u)$,

$$
\left[u^{2} g_{R}^{\prime \prime}(u)+u g_{R}^{\prime}(u)\right]\left[2 u g_{R}^{\prime}(u)+g_{R}(u)\right]-2 u^{2} g_{R}^{\prime \prime}(u)-u g_{R}^{\prime}(u)+g_{R}(u)=0 .
$$

Alternatively, if we choose $\eta^{R}=u g_{R}^{\prime}(u)$ as variable [see eq.(5.11]), we find from eq. (4.7) the first order non-linear differential equation

$$
\left[\eta^{R}+g_{R}\right] \frac{d g_{R}}{d \eta^{R}}+\eta^{R}\left[2 \eta^{R}+g_{R}-2\right]=0
$$

That is, the invariance of eq.(15.2) under the rescaling of the variable $u$ allows to reduce by one the order of the differential equation.

Eq.(5.1) has as regular solution around $u=0$,

$$
g_{R}(u)=\sum_{n=1}^{\infty} c_{n}^{R} u^{n} \quad \text { with } \quad c_{n}^{\text {sphere }}=\left(\frac{4 \pi}{3}\right)^{\frac{n-1}{3}} c_{n}^{R},
$$

and we can choose $c_{1}^{R}=1$.

By inserting eq.(5.3) into eq.(5.2) the following nonlinear recurrence relation for the $c_{n}^{R}$ coefficients is obtained:

$$
c_{n}^{R}=\frac{1}{(2 n+1)(n-1)} \sum_{s=1}^{n-1} c_{s}^{R} c_{n-s}^{R} s^{2}(2 n-2 s+1) \quad \text { for } \quad n \geq 2 .
$$

We find,

$$
c_{1}^{R}=1, c_{2}^{R}=\frac{3}{5}, c_{3}^{R}=\frac{51}{70}, c_{4}^{R}=\frac{373}{315}, c_{5}^{R}=\frac{14911}{6600}, c_{6}^{R}=\frac{2047}{429}, \ldots
$$

in agreement with eqs. (2.15), (2.16) and (2.18).

By evaluating numerically the $c_{n}^{R}$ from the recurrence relation eq.(5.4), we find for large $n$ :

$$
c_{n}^{R} \stackrel{n \geqq 1}{=} 0.309360 \ldots \frac{n^{-\frac{5}{2}}}{\left(u_{G C}\right)^{n}}\left[1+\mathcal{O}\left(\frac{1}{n}\right)\right] .
$$

Here, $u_{G C}=0.30034 \ldots$ is the radius of convergence of the series eq. (5.3. $)$. Therefore, $u=u_{G C}$ is the nearest singularity of $g_{R}(u)$ in the $u$-plane.

As we see from eq.(4.9),$g_{R}(u)$ gives the grand canonical partition function as a function of $u=t_{\eta} \eta^{R}$. Since $u=t_{\eta} \eta^{R}$ is proportional to the fugacity [see eq.(4.6)], $u_{G C}$ must be related to the critical point of the selfgravitating gas in the grand canonical ensemble. Indeed, using the critical value for $\eta^{R}$ in the grand canonical ensemble [2],

$$
\eta_{G C}^{R}=0.79735 \ldots \text { and } \quad f\left(\eta_{G C}^{R}\right)=\frac{2}{3 \eta_{G C}^{R}}
$$

from eq.4.8 we obtain,

$$
u_{G C}=\eta_{G C}^{R} t_{\eta_{G C}^{R}}=\eta_{G C}^{R} e^{-\eta_{G C}^{R}} f\left(\eta_{G C}^{R}\right)=\frac{2}{3} e^{-\eta_{G C}^{R}}=0.30034 \ldots
$$


in perfect agreement with the value for $u_{G C}$ in eq.(5.5). The large order behaviour of the expansion coefficients eq.(5.5) corresponds to a $\left(u_{G C}-u\right)^{\frac{3}{2}}$ behaviour of the function $g_{R}(u)$. More precisely, for $u \rightarrow u_{G C}$ from eq.(5.2) we find,

$$
g_{R}(u) \stackrel{u \rightarrow u_{G C}}{=} 2\left(1-\eta_{G C}^{R}\right)+\eta_{G C}^{R}\left(\frac{u}{u_{G C}}-1\right)+\frac{2}{3} \sqrt{2-\eta_{G C}^{R}}\left(1-\frac{u}{u_{G C}}\right)^{\frac{3}{2}}+\mathcal{O}\left[\left(u-u_{G C}\right)^{2}\right] .
$$

Expanding this asymptotic behaviour in powers of $u$ yields,

$$
g_{R}(u) \stackrel{u \rightarrow u_{G C}}{=} 2\left(1-\eta_{G C}^{R}\right)+\eta_{G C}^{R}\left(\frac{u}{u_{G C}}-1\right)+\frac{1}{2} \frac{\sqrt{2-\eta_{G C}^{R}}}{\pi} \sum_{k=0}^{\infty} \frac{\Gamma\left(k-\frac{3}{2}\right)}{k ! u_{G C}^{k}} u^{k} .
$$

This implies for the coefficients $c_{n}^{R}$ the following large order behaviour,

$$
c_{n}^{R} \stackrel{n \geqq 1}{=} \frac{1}{2} \sqrt{\frac{2-\eta_{G C}^{R}}{\pi}} \frac{n^{-\frac{5}{2}}}{\left(u_{G C}\right)^{n}}\left[1+\mathcal{O}\left(\frac{1}{n}\right)\right] .
$$

which exactly coincides with eq.(5.5) since,

$$
\frac{1}{2} \sqrt{\frac{2-\eta_{G C}^{R}}{\pi}}=0.309360 \ldots
$$

In summary, the investigation here on the cluster expansion for the selfgravitating gas shows:

- The selfgravitating gas admits a consistent thermodynamic limit $N, V \rightarrow \infty$ with $\frac{N}{V^{\frac{1}{3}}}$ fixed. In this limit, extensive thermodynamic quantities like energy, free energy, entropy are proportional to $N$. That is, in this limit the energy, free energy, and entropy per particle are well defined and finite.

- The cluster expansion and the mean field approach provide the same results in the thermodynamic limit $N, V \rightarrow \infty$ with $\frac{N}{V^{\frac{1}{3}}}$ fixed.

- The partition function needs a small short-distance cutoff $a$ in order to be well defined, the thermodynamic limit has a finite limit for $a \rightarrow 0$. In other words, the contributions to the partition function that diverge for $a \rightarrow 0$ are subdominant for $N \rightarrow \infty$ [see eq.(2.19)]. That is, the $N, V=\infty$ limit of the cutoff model (with $\frac{N}{V^{\frac{1}{3}}}$ fixed) has a finite limit for $a \rightarrow 0$.

\section{THE STABILITY OF THE THERMODYNAMIC LIMIT $\mathbf{N}, \mathbf{V}=\mathrm{L}^{3} \rightarrow \infty$ WITH $\frac{\mathrm{N}}{\mathrm{L}}$ FIXED (FIXED $\eta$ )}

As shown in refs. 2] there are two phases in the selfgravitating gas: $\eta<\eta_{0}$ and $\eta>\eta_{0}$ where $\eta_{0}=1.51024 \ldots$ for spherical geometry and $\eta_{0} \simeq 1.515$ for cubic geometry. For $\eta>\eta_{0}$ the selfgravitating gas collapses into a extremely dense phase with large and negative pressure. For $\eta<\eta_{0}$ the selfgravitating gas is perfectly stable. The mean field applies in this gaseous phase and coincides with the expansion in powers of $\eta$ discussed in the previous section. Such expansion converges within the gaseous phase. The variable $\eta$ is related to the Jeans' length of the system as

$$
\eta=3\left(\frac{L}{d_{J}}\right)^{2} \quad, \quad L=V^{\frac{1}{3}}
$$

where

$$
d_{J}=\sqrt{\frac{3 T}{m}} \frac{1}{\sqrt{G m \rho}} \quad, \quad \rho \equiv \frac{N}{V}
$$

The gas collapses in the canonical ensemble for $\eta>\eta_{0}$ which corresponds to $L \gtrsim d_{J}$. This corresponds to the Jeans' instability. 
The relevance of the ratio $\frac{G m^{2}}{V^{\frac{1}{3}} T}$ has been noticed on dimensional grounds $[9]$. However, the dimensionality argument alone cannot single out the crucial factor $N$ in the variable $\eta$. Notice that $\eta$ contains the ratio $\frac{N}{V^{\frac{1}{3}}}$ and not $\frac{N}{V}$. Therefore, in the thermodynamic limit

$$
V \sim N^{3} \quad \text { and } \quad \rho=\frac{N}{V} \sim \frac{1}{N^{2}} \rightarrow 0 .
$$

As $N, V \rightarrow \infty, \eta$ is kept fixed in the same way as the temperature $T$. The energy, the product $P V$, the free energy, the entropy are expressed as $N$ times functions of $\eta$. The chemical potential, the specific heats, the compressibilities are just functions of $\eta$.

In a recent e-print [] it was stated that the thermodynamic functions for the selfgravitating gas diverge in the thermodynamic limit $N, V \rightarrow \infty$ with $\frac{N}{V^{\frac{1}{3}}}$ fixed.

We show here below that the statements made in ref. [7] have crucial failures which invalidate the conclusions given in ref. [7].

Such statements in ref. [7] are based in the inequality [eq.(30) in ref. [7]],

$$
\mathcal{Z}_{\mathrm{C}} \geq \frac{1}{N !} \beta^{-3 N / 2} \int_{V_{0}^{N}} \prod_{i=1}^{N} d^{3} r_{i} \exp \left[-\beta \sum_{i<j} \phi_{i j}\right] \geq \frac{V_{0}^{N}}{N !} \exp \left[-\beta \sum_{i<j}\left\langle\phi_{i j}\right\rangle_{V_{0}}\right],
$$

where

$$
\left\langle\phi_{i j}\right\rangle_{V_{0}}=\frac{1}{V_{0}^{N}} \int_{V_{0}^{N}} \prod_{k=1}^{N} d^{3} r_{k} \phi_{i j}, \text { and } \phi_{i j} \equiv-\frac{G}{\left|\vec{r}_{i}-\vec{r}_{j}\right|},
$$

and the last inequality follows from the property of the exponential function: $\langle\exp (y)\rangle \geq \exp (\langle y\rangle)$.

To write such inequality the author of ref. 7] considers $N$ selfgravitating particles in a volume $V=R^{3}$ with $N \sim R$, that is, in the conditions of the above thermodynamic limit (i. e. $N / V^{1 / 3}$ fixed).

Then, a portion of such volume of linear size $R_{0}<R$ is considered: at this precise point the author assumes that $N \sim R_{0}^{3}$, that is, he assumes a extremely dense distribution of particles within the volume of size $R_{0}$.

But for large $N$ such distribution necessarily collapses since the gravitational gas avoids collapse only when $N \sim R_{0}$. In terms of the parameter $\eta$, the assumption $N \sim R_{0}^{3}$ implies

$$
\eta_{0} \sim N / R_{0} \sim\left(R_{0}\right)^{2} \gg 1
$$

which is deep in the collapsed phase [2]. That is, the assumption $N \sim R_{0}^{3}$ necessarily implies that the gas collapses. The subsequent statements made in ref. [7] are direct consequences of this assumption and are not valid.

It is clearly true that the partition function sums over all configurations including collapsed situations. However, these collapsed configurations have a negligible weight for $\eta<\eta_{0} \sim N / R_{0}$. The argument of ref. [7] applies only for $\eta>\eta_{0}$ and only for such values of $\eta$ collapsed states dominate the partition function. It must be noticed that in Nature, if collapsed configurations would always dominate selfgravitating systems, then, stars, galaxies and the interstellar medium would have collapsed since longtime. Fortunately, this argument in ref. [7] is not valid.

As stressed in refs [2, 3] there are two regimes for the selfgravitating gas in the canonical ensemble: $\eta<\eta_{0}$ and $\eta>\eta_{0}$ with $\eta_{0}=1.51024 \ldots$ for spherical geometry and $\eta_{C} \simeq 1.515$ for cubic geometry. For $\eta>\eta_{0}$ the selfgravitating gas do collapse into a extremely dense phase. It is to this collapsed phase and only to it that the eqs.(30)-(33) of ref. [7] apply.

The same comments applies to the convergence of the series expansions of the thermodynamic quantities discussed in ref. 7] [eq.(34) of ref. 7] above]. The series in powers of $\eta$ only converge for $\eta<\eta_{C}$. Actually, the calculation of the radius of convergence of such series in ref. [2, 3] provided us an independent check of the numerical value of $\eta_{C}$.

In the same token, the thermodynamic limit proposed in ref. [8] leads to a cataclysmic collapse for the selfgravitating gas. It is proposed in ref. [8] to take $N \rightarrow \infty$ with $V \sim \frac{1}{N} \rightarrow 0$.

It is obvious that in such limit the gas collapses since the volume vanishes. 


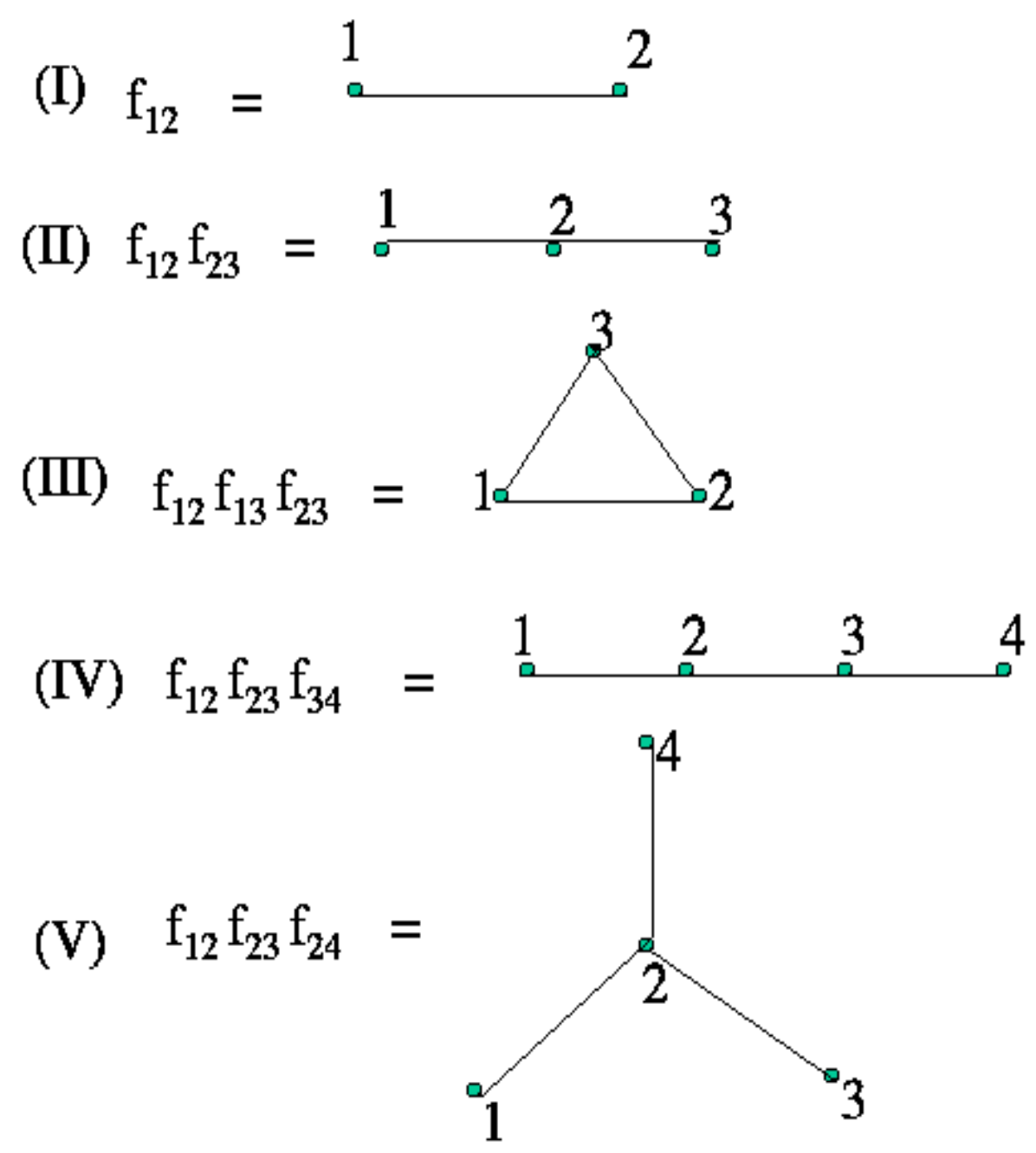

FIG. 1: Diagrams contributing to the cluster expansion. Diagram (I) gives $c_{2}$. Diagram (II) gives $c_{3}$. Diagrams (III), (IV) and (V) contribute to $c_{4}$ but (III) is subdominant for large $N$.

More precisely, that means $\eta \sim N^{\frac{4}{3}} \rightarrow+\infty$ deeply in the extremely dense phase. Furthermore, it is wrongly stated in sec. 4 of ref. [8] that the the entropy is not proportional to $N$ as in refs. [2, [3].

[1] R. Emden, Gaskugeln, Teubner, Leipzig und Berlin, 1907.

S. Chandrasekhar, 'An introduction to the Study of Stellar Structure', Chicago Univ. Press, 1939.

W. B. Bonnor, Mon. Not. R. astr. Soc. 116, 351 (1956).

R. Ebert, Z. Astrophys. 37, 217 (1955).

V. A. Antonov, Vest. Leningrad Univ. 7, 135 (1962).

D. Lynden-Bell and R Wood, Mon. Not. R. astr. Soc. 138, 495 (1968).

G. Horwitz and J. Katz, Ap. J. 211, 226 (1977) and 222, 941 (1978).

T. Padmanabhan, Phys. Rep. 188, 285 (1990).

W. C. Saslaw, 'Gravitational Physics of Stellar and Galactic Systems', Cambridge Univ. Press, 1987.

[2] H. J. de Vega, N. Sánchez, Nucl. Phys. B 625, 409 (2002).

[3] H. J. de Vega, N. Sánchez, Nucl. Phys. B 625, 460 (2002). 
[4] E. V. Votyakov, A. De Martino, D. H. E. Gross, Eur. Phys. J. B29, 593 (2002) and Nucl. Phys. B654, 427 (2003). P. H. Chavanis, I. Ispolatov, Phys. Rev. E66, 036109 (2002).

B. Leong, W. Saslaw, astro-ph/0308415

[5] H. J. de Vega, J. A. Siebert, Phys. Rev. E66, 016112 (2002).

[6] T. L. Hill, Statistical Mechanics, McGraw-Hill, New York, 1956.

[7] V. Laliena, astro-ph/0303301

[8] L. Velazquez, F. Guzmán, cond-mat/0205085 and 0303444.

[9] See the last reference under 1]. 